\title{
TOPICS IN THE THEORY OF MARKOFF CHAINS
}

\author{
BY
}

J. L. DOOB

Let $P(t):\left(p_{i j}(t)\right)$ be a matrix (finite- or infinite-dimensional), depending on $t>0$, whose elements satisfy the following conditions

$$
p_{i j}(t) \geqq 0, \quad \sum_{j} p_{i j}(t)=1, \quad P(s) P(t)=P(t) P(s)=P(s+t) .
$$

Then $p_{i j}(t)$ can be considered a transition probability of a Markoff chain: A system is supposed which can assume various numbered states, and $p_{i j}(t)$ is the probability that the system is in the $j$ th state at the end of a time interval of length $t$, if it was in the $i$ th state at the beginning of the interval. The present paper will be divided into two parts. In the first, the regularity properties of $P(t)$, and its asymptotic properties as $t \rightarrow 0, t \rightarrow \infty$ are studied. These problems have been solved in the finite-dimensional case by Doeblin( $\left.{ }^{1}\right)$. In the infinite-dimensional case new situations can arise, and the results are somewhat different. The method of approach is new, depending on two theorems (Theorems 2 and 3 ) concerning matrices whose elements are non-negative, and which have row sums less than or equal to 1 . The method of approach can also be applied to the study of the asymptotic properties of the powers of a matrix of non-negative elements, with row sums 1 . In the second part of the paper, the actual transitions connected with Markoff chains are investigated: That is, the properties of the function $\xi(t)$, the number of the state which the given system assumes at time $t$, are investigated. The continuity properties of $\xi(t)$ are analyzed, and related to the regularity properties of the $p_{i j}(t)$.

Lемма 1. Suppose that the function $f(t)$, defined for all $t>0$, satisfies the functional equation

$$
f(s+t)=\sum_{n} g_{n}(s) h_{n}(t) \quad(s, t>0),
$$

where $g_{n}(s), h_{n}(s)$ are defined for $s>0$, where $h_{n}(s)$ is measurable, and where for each fixed $s$, if $0<a<b$, the series converges uniformly for $a \leqq t \leqq b$. Then $f(t)$ is continuous, for all $t>0$.

Presented to the Society, December 31, 1941; received by the editors May 24, 1941.

(1) Bulletin des Sciences Mathématiques, (2), vol. 62 (1938), pp. 21-32, and vol. 63 (1939), pp. 35-37. In the following, these papers will be referred to as Doeblin (I). Fréchet has discussed the solutions of $(0.1)$ in great detail, in the finite-dimensional case, with full references to earlier authors in his book Traité du Calcul des Probabilités et de ses Applications, vol. 1, Part 3, Book 2, Méthode des fonctions arbitraires . . , Paris, 1938. 
It will be sufficient to prove that if a value $t_{0}$ of $t$ is given, and if $\left\{\delta_{j}\right\}$ is a sequence of numbers approaching 0 , then $f\left(t_{0}+\delta_{a_{j}}\right) \rightarrow f\left(t_{0}\right)$, for some subsequence $\left\{\delta_{a_{j}}\right\}$ of $\left\{\delta_{j}\right\}$. By a theorem of Auerbach $\left({ }^{2}\right)$, there is, corresponding to each $h_{n}(t)$, a subsequence $\left\{\delta_{a_{j}}\right\}$ of $\left\{\delta_{j}\right\}$, such that

$$
\lim _{j \rightarrow \infty} h_{n}\left(t+\delta_{a_{j}}\right)=h_{n}(t)
$$

for almost all $t$ in the interval $0<t<t_{0}$. There is then, using the diagonal process, a subsequence $\left\{\delta_{a_{j}}\right\}$ of $\left\{\delta_{j}\right\}$ such that (1.2) is true for all $n, 0<t<t_{0}$, except possibly on a $t$-set of measure 0 . If $0<t<t_{0}$, and if $j$ is large,

$$
f\left(t_{0}+\delta_{a j}\right)=\sum_{n} g_{n}\left(t_{0}-t\right) h_{n}\left(t+\delta_{a_{j}}\right),
$$

and if $t$ is not in the exceptional set, (1.3) implies, when $j \rightarrow \infty$,

$$
f\left(t_{0}+\delta_{a_{j}}\right) \rightarrow \sum_{n} g_{n}\left(t_{0}-t\right) h_{n}(t)=f\left(t_{0}\right)
$$

(because of the uniform convergence of the series in (1.3) with respect to $j$ ), as was to be proved.

THEOREM 1. If the matrix function $P(t)$ satisfies (0.1), the measurability of the $p_{i j}(t)$ implies their continuity.

This follows at once from Lemma 1. It has been shown by Doeblin (I) and it will be a corollary of results to be proved below, that the $p_{i j}(t)$ satisfying $(0.1)$ are always continuous if the matrix $P(t)$ is finite-dimensional, even if measurability is not assumed. The following example shows that there are non-measurable solutions of $(0.1)$.

In this example, the $p_{i j}(t)$ take on only the values 0,1 , and $P(t)$ is a permutation matrix. Hamel has shown that there is a function $f(x)$, defined for all real $x$, taking on only rational values, and satisfying the functional equation $\left(^{3}\right) f(x+y)=f(x)+f(y)$. Let $\left\{r_{n}\right\}$ be an enumeration of all the rational numbers, and let $T_{s}$ be the transformation of these numbers taking $r_{j}$ into $r_{j}+f(s)$. The transformation can be represented by a matrix $P(s):\left(p_{i j}(s)\right)$, where $p_{i j}(s)=1$ if $T_{s} r_{i}=r_{j}$, and $p_{i j}(s)=0$ otherwise. Then evidently $P(s+t)$ $=P(s) P(t)$, and $(0.1)$ is satisfied. The functions $p_{i j}(t)$ are not measurable, since they obviously are not continuous.

The following theorem describes completely the solutions of $(0.1)$ which are independent of $t$. It will be useful to weaken (0.1) slightly. The theorem is essentially known, at least in an indirect form $\left(^{4}\right)$.

(2) Fundamenta Mathematicae, vol. 11 (1928), pp. 196-197.

(3) Mathematische Annalen, vol. 60 (1905), pp. 459-462. To ensure that Hamel's $f(x)$ take on only rational values, we can set, using his notation, $f(a)=1, f(b)=\cdots=0$.

(4) Cf., for example, K. Yosida and S. Kakutani, Japanese Journal of Mathematics, vol.16 (1939), pp. 47-55. 
Theorem 2. Let $U:\left(u_{i j}\right)$ be a matrix of elements satisfying the following conditions:

$$
u_{i j} \geqq 0, \quad \sum_{j} u_{i j} \leqq 1, \quad U^{2}=U .
$$

Then the subscripts can be divided into mutually exclusive classes $\left(^{(5)} F, G_{1}, G_{2}, \ldots\right.$ such that

(a) $u_{i j}=0$, if $j \in F$;

(b) there are positive numbers $u_{j}, j \notin F$, such that ${ }^{6}$ )

$$
\begin{array}{crl}
u_{i j} & =\delta_{I J} u_{j}, & \text { (if } i \in I, j \in J), \\
\sum_{j \in J} u_{i j}=\sum_{j \in J} u_{j}=1 & (i \in I=J) ;
\end{array}
$$

(c) there are non-negative numbers $\left\{\rho_{i J}\right\}$ such that

$$
u_{i j}=\rho_{i J} u_{j}
$$

Conversely, if the $u_{i j}$ satisfy (a), (b), (c), then (2.1) is true.

Suppose (2.1) is true. Define $F$ as the set of integers $j$ with $p_{i j}=0$ for all $i$. Then (a) is true. Unless $U$ is the null matrix, there will be subscripts not in $F$. The extreme members of the inequality

$$
\sum_{k} u_{i k}=\sum_{j, k} u_{i j} u_{j k}=\sum_{j} u_{i j}\left(\sum_{k} u_{j k}\right) \leqq \sum_{j} u_{i j}
$$

are equal; so if $\sum_{k} u_{j k}<1, u_{i j}=0: j \in F$. Let $\xi_{1}, \xi_{2}, \cdots$ be any numbers satisfying the conditions:

$$
\sum_{i}\left|\xi_{j}\right|<\infty, \quad \sum_{i} \xi_{i} u_{i j}=\xi_{j}
$$

Then $\xi_{j}=0$ if $j \in F$. If $G$ is the set of integers $j$ for which $\xi_{j}>0$,

$$
\sum_{j \in G} \xi_{j}=\sum_{j \in G} \sum_{i} \xi_{i} u_{i j} \leqq \sum_{i, j \in G} \xi_{i} u_{i j} \leqq \sum_{i \in G} \xi_{i},
$$

and there is an impossible inequality unless $u_{i j}=0$ whenever $\xi_{i}<0, \xi_{j}>0$. Let $i, j$ be any two distinct integers not in $F$. Unless the $i$ th and $j$ th columns of $U$ are proportional (neglecting elements in the columns whose first subscripts are in $F)$, there are integers $r, s(\notin F)$, such that

(5) The class $F$ may be absent, or the $G_{\nu}$ may be absent. The latter case will arise when and only when $U$ is the null matrix.

(6) In the following, capital letters $I, J, K$ will be used to denote the $G_{\nu}$, and a subscript $i$ will always belong to the class $I$, and so on, unless the contrary is explicitly stated. The notation $\delta_{I J}$ is the usual Kronecker $\delta$. 


$$
\left|\begin{array}{ll}
u_{r i} & u_{r i} \\
u_{s i} & u_{s i}
\end{array}\right| \neq 0
$$

Then $\lambda, \mu$ can be chosen so that $\lambda u_{r i}+\mu u_{s i}<0, \lambda u_{r j}+\mu u_{s j}>0$. Since $\xi_{k}=\lambda u_{r k}+\mu u_{s k}$ provides a solution of (2.3) with $\xi_{i}<0, \xi_{j}>0$, it follows that $u_{i j}=0$ if (2.5) is true. The subscripts not in $F$ fall into classes, $G_{1}, G_{2}, \cdots$, putting subscripts in the same class if the corresponding partial columns are proportional; if $i, j(\notin F)$ are not in the same class, $u_{i j}=0$. The elements $u_{i j}$ with $i$ in a class $G_{\nu}$ determine a matrix of rank 1 . The rows of this matrix are therefore proportional, in fact identical, since the row sums are 1 . We can thus write $u_{i j}=\delta_{I J} u_{j}(i \in I, j \in J)$, and

$$
\sum_{j \in J} u_{j}=\sum_{j} u_{j_{0} j}=1 \quad\left(j_{0} \in J\right) .
$$

If $i \in F, j \in J$,

$$
u_{i j}=\sum_{k} u_{i k} u_{k j}=\left(\sum_{k \in J} u_{i k}\right) u_{j}=\rho_{i J} u
$$

where $\rho_{i}$ is defined by the sum in the parentheses. If $j \notin F, u_{j}$ cannot vanish, since the elements of the $j$ th column $(j \notin F)$ cannot all vanish. We have now shown that (2.1) implies (a), (b), (c). Conversely, if (a), (b), (c) are true, (2.1) can be checked at once.

THEOREM 3. Let $\mathfrak{M}$ be a set of matrices (finite-or infinite-dimensional) with non-negative elements, and row sums less than or equal to 1 . Suppose that the matrices in $\mathfrak{M}$ form a group $\mathfrak{M}^{\prime}$. There is then a $U \in \mathfrak{M}$ (the identity in $\mathfrak{M}^{\prime}$ ) with $U^{2}=U$. If $U$ is the null matrix, $U$ is the only matrix in $\mathfrak{M}$, and $\mathfrak{M}^{\prime}$ consists only of the identity. If $U$ is not the null matrix, we shall use the notation of Theorem 2 to describe its elements. The group $\mathfrak{M}^{\prime}$ is always isomorphic to a permutation group acting on the $G_{\nu}$. If $\left(p_{i j}\right)$ is a matrix of $\mathfrak{M}$, and if the corresponding permutation takes $I_{1}$ into $I_{2}\left({ }^{7}\right)$, then

$$
\begin{array}{lr}
p_{i j}=\delta_{I_{2} J} u_{j} & \left(i \in I_{1}, j \in J\right), \\
p_{r i}=\rho_{r I_{1}} u_{i} & \left(r \in F, i \in I_{2}\right), \\
p_{i j}=0 & (j \in F) .
\end{array}
$$

Evidently if $U$ is the null matrix, it is the only matrix in $\mathfrak{M}$, and $\mathfrak{M}^{\prime}$ consists only of the identity. We shall assume from now on that $U$ is not the null matrix, and use the notation of Theorem 2. Suppose that $P:\left(p_{i j}\right) \in \mathfrak{M}$. Then since $P=P U=U P$,

(7) As usual, letters $I, J$ refer to the $G_{\nu}$. 


$$
p_{i k}=\sum_{j} p_{i j} u_{j k}=\sum_{j} u_{i j} p_{j k} .
$$

If $k \in F,(3.2)$ shows that $p_{i k}=0$, the last equation of (3.1). If $i \in I, k \in K$, (3.2) becomes

$$
\begin{aligned}
p_{i k} & =\left(\sum_{r \in K} p_{i r}\right) u_{k}, \\
p_{i k} & =\sum_{r \in \in_{K}} u_{r} p_{r k} .
\end{aligned}
$$

According to $\left(3.2^{\prime}\right), p_{i k} / u_{k}$ depends only on $i, K$, and according to (3.2'), $p_{i k}$ depends only on $I, k$. Then $p_{i k} / u_{k}$ depends only on $I, K$ :

$$
p_{i k}=\sigma_{I K} u_{k} \quad(i \in I, k \in K) .
$$

There is a $P^{\prime}:\left(p_{i j}^{\prime}\right)$ in $\mathfrak{M}$ which is the inverse of $P$ in $\mathfrak{M}^{\prime}$. If we write $p_{i k}^{\prime}=\sigma_{I K}^{\prime} u_{k}$, for $i, j \notin F$, the equation $U=P^{\prime} P$ implies

$$
u_{i k}=\delta_{I K} u_{k}=\sum_{J} \sigma_{I J}^{\prime} \sigma_{J K} u_{k} .
$$

The $\sigma_{I J}, \sigma_{I J}^{\prime}$ are non-negative and

$$
\sum_{J} \sigma_{I J}^{\prime}=\sum_{k} p_{i k}^{\prime} \leqq 1
$$

If $I=K$ in (3.4), we obtain

$$
1=\sum_{J} \sigma_{I J}^{\prime} \sigma_{J I} \leqq \sum_{J} \sigma_{I J}^{\prime} \leqq 1 .
$$

There must be equality throughout in (3.6); therefore if $\sigma_{J I}<1$, it follows that $\sigma_{I J}^{\prime}=0$. The matrices $\left(\sigma_{I J}\right),\left(\sigma_{I J}^{\prime}\right)$ play symmetric roles; so if $\sigma_{J I}^{\prime}<1$, it follows that $\sigma_{I J}=0$. Then if $\sigma_{I J}<1, \sigma_{J I}^{\prime}=0<1$; so $\sigma_{I J}=0$. Each element in the matrix $\left(\sigma_{I J}\right)$ is either 1 or 0 , and by (3.6) there is a 1 in each row of $\left(\sigma_{I J}^{\prime}\right)$ and therefore in each row of $\left(\sigma_{I J}\right)$. If $\sigma_{I_{1} I_{2}}=1$, the matrix $\left(\sigma_{I J}\right)$ defines the permutation of the $G_{\nu}$ taking $I_{1}$ into $I_{2}$. The matrix $\left(\sigma_{I J}^{\prime}\right)$ defines the inverse of this permutation. Equation (3.3) becomes the first equation of (3.1), equation (3.2) implies the second equation of (3.1), and the third equation of (3.1) has already been verified. The equations of (3.1) induce an isomorphism between the permutations defined by the $\left(\sigma_{I J}\right)$ permutation matrices and $\mathfrak{M}^{\prime}$.

Corollary 1. Suppose in Theorem 3 that $\mathfrak{M}$ contains its limit matrices $\left.{ }^{8}\right)$. Then the corresponding permutation group on the $G_{\nu}$ has the property that each $G_{j}$

(8) The matrices $\left\{M^{(n)}:\left(m_{i j}^{(n)}\right)\right\}$ will be said to converge to $M:\left(m_{i j}\right), M^{(n)} \rightarrow M$, if $m_{i j}^{(n)} \rightarrow m_{i j}$ for all $i, j$. The limit matrices of $\mathfrak{M}$ are matrices which are limits of convergent sequences of matrices in $\mathfrak{M}$. 
can go only into a finite number of the $G_{\nu}$. If in addition it is supposed that corresponding to each $A \in \mathfrak{M}$ and positive integer $n$ there is a $B \in \mathfrak{M}$ such that $B^{n}=A$, then $\mathfrak{M}$ consists of only a single matrix, of the type described in Theorem 2 .

Suppose that $\mathfrak{M}$ contains its limiting matrices, and that some $G_{\nu}$, say $G_{\alpha}$, goes into infinitely many $G_{\nu}$ under the permutations of the group. Then there is a limiting matrix $\left(p_{i j}\right)$ of $\mathfrak{M}$ such that $p_{i j}=0$ if $i \in G_{\alpha}$. But a matrix with these rows of zeros cannot be in $\mathfrak{M}$, so $G_{\alpha}$ cannot have the supposed property. The first part of the corollary is thus proved. Now suppose both hypotheses of the corollary are satisfied. It will be sufficient to prove that the group of permutations on the $G_{\nu}$ is the identity. Let $G_{\alpha}$ be any $G_{\nu}$. We have already shown that $G_{\alpha}$ can go only into a finite number of $G_{\nu}$, say $G_{a_{1}}, \cdots, G_{a_{j}}$, under the permutations of the group. The permutations then permute $G_{a_{1}}, \cdots, G_{a_{j}}$ among themselves, and any element of the group of permutations on $G_{a_{1}}, \cdots, G_{a_{j}}$ has order a factor of $j$ !. But any element in this group of permutations is by hypothesis the $j$ !th power of some other element; it must therefore be the identity. Then $j=1$, and $G_{\alpha}$ is transformed into itself by every permutation of the group, as was to be proved.

Corollary 2. Any matrix function $P(t):\left(p_{i j}(t)\right)$ with measurable elements $p_{i j}(t)$ satisfying $(0.1)$ for all $t$ (including 0 and negative values) is independent of $t: P(t) \equiv U$, where $U$ has the properties described in Theorem 2.

We can assume that some $P(t)$ is not the null matrix, or there would be nothing to prove. The matrices $P(t)$ form a family $\mathfrak{M}$ satisfying the conditions of Theorem 3. Moreover each $p_{i j}(t)$ is continuous, if $t>0$, by Theorem 1 , and so for all $t$, from (0.1). Using the notation of Theorem 3 , if $i \notin F, p_{i j}(t)=u_{j}$ or $p_{i j}(t)=0$. Then if $i \notin F, p_{i j}(t)$ is independent of $t$. This means that $\mathfrak{M}^{\prime}$ consists only of the identity, so $P(t)$ is independent of $t: P(t) \equiv U$. The example above shows that the measurability of the $p_{i j}(t)$ is a necessary part of the hypotheses.

THEOREM 4. If the $p_{i j}(t)$ satisfying (0.1) are continuous, then $\lim _{t \rightarrow 0} P(t)=U$ exists. The matrix $U$ is a non-null matrix of the type described in Theorem 2, and $\left.{ }^{9}\right)$

$$
U P(t) \leqq P(t) U=P(t) .
$$

(In the following we shall use the notation of Theorem 2.) Moreover

$$
p_{i j}(t)=0
$$

$(j \in F)$.

There are continuous functions $\Pi_{I J}(t)$, satisfying $(0.1)$ and

( ${ }^{9}$ An inequality between two matrices is defined to mean the same inequality between their corresponding elements. 


$$
\lim _{t \rightarrow 0} \Pi_{I J}(t)=\delta_{I J}
$$

such that

$$
p_{i j}(t)=\Pi_{I J}(t) u_{j}
$$

There are continuous functions $\Pi_{i J}(t)(i \in F)$ such that $\left({ }^{10}\right)$

$$
\begin{aligned}
p_{i j}(t) & =\Pi_{i J}(t) u_{j} & \\
\Pi_{i K}(t) & \geqq \sum_{J} \rho_{i J} \Pi_{J K}(t) . &
\end{aligned}
$$

Conversely, if the $p_{i j}(t)$ satisfy (0.1) and if $\lim _{t \rightarrow 0} P(t)$ exists, the $p_{i j}(t)$ are continuous.

Neglecting subscripts in $F$, this theorem reduces the study of $P(t)$ to that of $\left(\Pi_{I J}(t)\right)$ in which case the limit matrix $(t \rightarrow 0)$ is the identity.

Let $U:\left(u_{i j}\right), U^{\prime}:\left(u_{i j}^{\prime}\right)$ be limiting matrices of $P(t), t \rightarrow 0$. Then $(0.1)$ implies (4.1). The equal $i$ th row sums in (4.1) are

$$
\sum_{j} p_{i j}(t) \sum_{k} u_{j k}=\sum_{k} p_{i k}(t)=1 .
$$

Since the row sums of $U$ are less then or equal to $1,(4.6)$ implies that if $\sum_{k} u_{j k}<1, p_{i j}(t)=0$. Then in this case $u_{i j}=u_{i j}^{\prime}=0$ also. It follows from (4.1) that

$$
\sum_{j} u_{i j}^{\prime} u_{j k} \leqq u_{i k}^{\prime} \quad\left(U^{\prime} U \leqq U^{\prime}\right)
$$

Summing over $k$, since $u_{i j}^{\prime}=0$ if $\sum_{k} u_{j k}<1$, we see that both sides of (4.7) have $\operatorname{sum} \sum_{k} u_{i k}^{\prime}$; so there is equality in (4.7):

$$
U^{\prime} U=U^{\prime} \text {. }
$$

Replacing $U$ by $U^{\prime}$ in the inequality $U P(t) \leqq P(t)$, and letting $t$ approach 0 in such a way that $P(t) \rightarrow U$, we obtain

$$
U^{\prime} U \leqq U
$$

Then combining $\left(4.7^{\prime}\right)$ and (4.8), we have $U^{\prime} \leqq U$, and by symmetry $U \leqq U^{\prime}$; so $U=U^{\prime}$. There is thus only one limiting matrix $U: P(t) \rightarrow U$. Since equation (4.7') becomes $U^{2}=U$, Theorem 2 is applicable. In the following, we shall use the notation of that theorem. If $k \in F, u_{j k}=0$; therefore (using (4.1)) $p_{i k}(t)=0$ also, for all $i$. Then $U$ is not the null matrix. If $i, k \notin F,(4.1)$ implies

$$
\left(\sum_{j \in K} p_{i j}(t)\right) u_{k}=p_{i k}(t) \quad(k \in K),
$$

(10) If the $G_{\nu}$ contain only one subscript each, so that $p_{i j}(t) \rightarrow \delta_{i j}(t \rightarrow 0)$ if $i \notin F$, then we can $\operatorname{read} p_{i j}(t)$ for $\Pi_{I J}(t), p_{i j}(t)$ for $\Pi_{I J}(t)$ throughout. 
and

$$
\sum_{j \in I} u_{j} p_{j k}(t) \leqq p_{i k}(t)
$$

There is equality in (4.10) (and we shall refer to it as if it were so written), because summing over $k$ gives 1 on both sides. Equations (4.9) and (4.10) imply that if $i, k \in F, p_{i k}(t) / u_{k}$ depends only on $I, K: p_{i k}(t)=\Pi_{I K}(t) u_{k}$. Evidently the matrix function $\left(\Pi_{I J}(t)\right)$ satisfies $(0.1)$, and (4.3) is true. If $i \in F$, and $k \notin F$, (4.1) implies

$$
\sum_{J} \rho_{i J} \Pi_{J K}(t) \leqq\left(\sum_{j \in K} p_{i j}(t)\right) u_{k}=p_{i k}(t),
$$

so that if $\Pi_{i K}(t)$ is defined as the parentheses in (4.11), (4.5) follows at once.

Conversely, if $P(t) \rightarrow U(t \rightarrow 0)$,

$$
\lim _{t \downarrow 0} P(s+t)=P(S) U
$$

The function $p_{i j}(t)$ having a right-hand limit for all $t$ has at most denumerably many discontinuities, is therefore measurable, and continuous (Theorem 1).

Theorem 5. Let $\alpha$ be a given subscript. Then if $P(t)$ satisfies $(0.1), p_{\alpha j}(t)$ will be continuous and $\lim _{t \rightarrow 0} p_{\alpha j}(t)$ will exist, for all $j$, if $\sum_{j} p_{\alpha j}(t)$ converges uniformly in some interval $0<t<t_{0}$.

Doeblin (I) proved that if $P(t)$ satisfies $(0.1)$ and is finite-dimensional, then the $p_{i j}(t)$ are continuous and have unique limits as $t \rightarrow 0$. This fact which evidently is a consequence of Theorem 5 , can be proved directly as follows. Let $\mathfrak{M}$ be the set of limiting matrices of $P(t), t \rightarrow 0$. Then $\mathfrak{M}$ satisfies the conditions of Theorem 3 , Corollary 1 , so $\mathfrak{M}$ contains only a single matrix $U$. It follows that $P(t) \rightarrow U$, and the $p_{i j}(t)$ are then continuous, by Theorem 4 .

Proof of Theorem 5. Let $G$ be the set of subscripts $\alpha$ with the property described in the theorem. The equation $P(s) P(t)=P(t) P(s)$ implies that if $A:\left(a_{i j}\right)$ is a limiting matrix of $P(t), t \rightarrow 0$, then

$$
\sum_{j} a_{i j} p_{j k}(t) \leqq \sum_{j} p_{i j}(t) a_{j k} .
$$

If $i \in G$, then $\sum_{j} a_{i j}=1$, and the sum over $k$ on the left is 1 , so that on the right is also 1 . Then there is equality in (5.1):

$$
\sum_{j} a_{i j} p_{j k}(t)=\sum_{j} p_{i j}(t) a_{j k}
$$

If $\sum_{k} a_{j k}<1$, then $p_{i j}(t)=0$, or the sum over $k$ on the right in $\left(5.1^{\prime}\right)$ would not be 1 . If $j \notin G$, we can find an $A$ with $\sum_{k} a_{j k}<1$, whence it follows that 
$p_{i j}(t)=0$ if $i \in G, j \notin G$. Let $P^{\prime}(t)$ be the matrix obtained by dropping all elements of $P(t)$ with a subscript not in $G$. Then $P^{\prime}(t)$ satisfies $(0.1)$ and has the property that any limiting matrix $(t \rightarrow 0)$ has row sums 1 . The proof given above of Doeblin's result goes through word for word, applied to $P^{\prime}(t)$. We have thus proved that $P_{i j}(t)$ is continuous, and $\lim _{t \rightarrow 0} p_{i j}(t)$ exists, if $\alpha \in G$, and in addition that $p_{i j}(t) \equiv 0$ if $\alpha \in G, j \notin G$.

We now turn to an examination of the limiting matrices of $P(t)$, as $t \rightarrow \infty$.

Theorem 6. Define the matrix $U:\left(u_{i j}\right)$ by

$$
\liminf _{t \rightarrow \infty} p_{i j}(t)=u_{i j}
$$

\section{Then}

(a) $U$ is a limiting matrix of $P(t)$, as $t \rightarrow \infty$; $U$ has the properties described in Theorem 2, and $P(t) U=U P(t)=U$;

(b) (6.1) can be sharpened to

$$
\lim _{t \rightarrow \infty} p_{i j}(t)=u_{i j}
$$

if $i$ is a subscript such that $\sum_{j} u_{i j}=1\left({ }^{11}\right)$.

(c) Using the notation of Theorem 2, and assuming that $U$ is not the null matrix,

$$
\begin{array}{rlrl}
p_{i j}(t) & =0 \\
\sum_{r \in J} u_{r} p_{r j}(t) & =u_{j} & & (i \in I, j \in I), \\
\sum_{j \in K} p_{i j}(t)+\sum_{j \in F} p_{i j}(t) \rho_{j K} & =\rho_{i K} & (j \in J),
\end{array}
$$

If $i \notin F$ or if $j \notin F\left(6.1^{\prime}\right)$ is true. If $i \notin F, p_{i j}(t)$ is continuous, and $\lim _{t \rightarrow 0} p_{i j}(t)$ exists. Moreover

$$
\lim _{t \rightarrow \infty} \sum_{r \in K} p_{i r}(t)=\rho_{i K}, \quad \lim _{t \rightarrow \infty} \sum_{j \in F} p_{i j}(t) \rho_{j K}=0 \quad(i \in F) .
$$

The fact that if $P(t)$ is finite-dimensional $\left(6.1^{\prime}\right)$ is always true, which follows from Theorem 6 , can be proved directly as follows. The set of limiting matrices (in this case) of $P(t), t \rightarrow \infty$, is seen at once to have the properties required in Theorem 3, Corollary 1 , so there is only one limiting matrix $U$ : $P(t) \rightarrow U$. This argument breaks down in the infinite-dimensional case, in which a more detailed analysis is necessary.

Let $\&$ be the class of limiting matrices $\left(a_{i j}\right)$ of $P(t)$, as $t \rightarrow \infty$. Then $\&$ includes all its limit matrices. This implies that $\mathfrak{R}$ contains one or more matrices minimizing $\sum_{i, j} 2^{-i} a_{i j}$. Let $\mathfrak{M}$ be the class of these minimal matrices. We shall show that $\mathfrak{M}$ contains only one matrix, $U$, defined by (6.1). The proof will be carried through in several steps.

(11) It follows that if the matrices are finite-dimensional, $\left(6.1^{\prime}\right)$ is true for all $i, j$, a fact due to Doeblin (I). 
(i) If $A \in \mathbb{R}$, then $P(t) A \in \mathbb{R}$.

This can be deduced at once from $(0.1)$.

(ii) If $A \in \mathfrak{M}$, then $A P(t)=P(t) A \in \mathfrak{M}$.

This follows, for $(0.1)$ implies that if $A:\left(a_{i j}\right) \in \mathfrak{M}$, there is a $B:\left(b_{i j}\right) \in \mathbb{R}$, depending on $t$ and on $A$, such that

$$
\sum_{j} b_{i j} p_{j k}(t) \leqq \sum_{j} p_{i j}(t) b_{j k}=a_{i k} .
$$

Summed over $k$, this means that

$$
\sum_{j} b_{i j} \leqq \sum_{k} a_{i k} \quad(i=1,2, \cdots)
$$

and only equality is possible, since $A \in \mathfrak{M}$. Then (6.4) becomes the equality $B P(t)=P(t) B=A$. Therefore,

$$
A P(t)=P(t) B P(t)=P(t) A .
$$

By (i), $P(t) A \in \mathbb{R}$, and summing over the $i$ th row of $P(t) A=A P(t)$ gives $\sum_{j} a_{i j}$, so $P(t) A \in \mathfrak{M}$, since $A \in \mathfrak{M}$.

(iii) If $A, B \in \mathfrak{M}$, then $A B=B A \in \mathfrak{M}$.

By (ii), if $A \in \mathfrak{M}$, it follows that $A P(t) \in \mathfrak{M}$. If $B \in \mathfrak{M}, A B$ is a limiting matrix of $A P(t), t \rightarrow \infty$, so $A B \in \mathfrak{M}$ since $\mathfrak{M}$ is closed. Moreover by. (ii), $A P(t)=P(t) A$, so $A B \geqq B A$. By symmetry, the reverse inequality is also true; so $A B=B A$.

(iv) If $A \in \mathbb{R}$ there is an $A^{\prime} \in \mathfrak{M}$ with $A^{\prime} \leqq A$.

For if $A \in \mathbb{R}$, there is, using (0.1), a $B \in \mathfrak{M}$ and a $C \in \mathbb{R}$ such that $A \geqq B C$, and since $B C \in \mathfrak{M}$ (as a limit of $B P(t) \in \mathfrak{M}$ ) this is the desired inequality.

(v) If $A, B \in \mathfrak{M}$, there is a $C \in \mathfrak{M}$ with $A=B C$.

We see this, for there is certainly, using (0.1), a $C \in \mathbb{R}$ with $A \geqq B C$. As we have seen, $B C \in \mathfrak{M}$, so there must be equality.

(vi) If $A \in \mathfrak{M}$, and if $n$ is any positive integer, there is a $B \in \mathfrak{M}$ such that $A=B^{n}$.

For, since $P(t / n)^{n}=P(t)$, if $A \in \mathfrak{M}$, there is a $B_{1} \in \mathbb{R}$ such that $B_{1}^{n} \leqq A$. By (iv) there is then a $B \in \mathfrak{M}$ with $B^{n} \leqq B_{1}^{n} \leqq A$. To show that there must be equality, we need only show that $B^{n} \in \mathbb{R}$. Since $B \in \mathfrak{M}, B B=B^{2} \in \mathfrak{M}$ by (iii). Then $B B^{2}=B^{3} \in \mathfrak{M}$, and so on.

Now (iii) and (v) imply that the matrices of $\mathfrak{M}$ form a commutative group The fact that $\mathfrak{M}$ is closed and that (vi) is true shows that $\mathfrak{M}$ has the properties required in Theorem 3 , Corollary 1 . There can therefore be only a single matrix $U:\left(u_{i j}\right)$ in $\mathfrak{M}$, and $U$ has the properties described in Theorem 2 . Because of (iv), $\lim \inf _{t \rightarrow \infty} p_{i j}(t)=u_{i j}$. From now on we shall assume the notation of Theorem 2. The equality $P(t) U=U P(t)=U$ follows from (ii). If $\sum_{j} u_{\alpha j}=1$, no limiting value of $p_{\alpha j}(t), t \rightarrow \infty$, can be greater than $u_{\alpha j}$, or there would be a limiting row having a sum greater than 1 . Then if $\sum_{j} u_{\alpha j}=1, \lim _{t \rightarrow \infty} p_{\alpha j}(t)=u_{\alpha j}$, for all $j$. In particular, $\left(6.1^{\prime}\right)$ is true if $i \notin F$. The equations of (6.2) are equiva- 
lent to the equations $P(t) U=U P(t)=U$. If $\left(a_{i j}\right)$ is a limiting matrix of $P(t)$ as $t \rightarrow 0,(6.2)$ implies that

$$
\sum_{r \in J} u_{r} a_{r j}=u_{i} \quad(j \in J) .
$$

Summing (6.6) over $j \in J$ we obtain

$$
\sum_{r \in J} u_{r}\left(\sum_{j \in J} a_{r j}\right)=\sum_{j \in J} u_{j}=1
$$

Then $\sum_{j} a_{r j}=1$ if $r \in J$. This implies that $\sum_{j} p_{r j}(t)$ converges uniformly in some interval $0<t<t_{0}$; so according to Theorem $5, p_{r j}(t)$ is continuous, and has a unique limit as $t \rightarrow 0$, if $r \in J$. Then this is true for any subscript $r \notin F$. As $t \rightarrow \infty$ in the last equation of (6.2) the first sum has an inferior limit greater than or equal to $\rho_{i K}$. Then there must actually be convergence; the first equation of (6.3) is true. The second sum in the last equation of (6.2) must then approach 0 ; (6.3) is true. Equation (6.3) is impossible, since $\lim \inf _{t \rightarrow \infty} p_{i k}(t)=\rho_{i K} u_{k}$, $(i \in F, k \in K)$ unless $p_{i k}(t) \rightarrow \rho_{i K} u_{k}$; so $\left(6.1^{\prime}\right)$ is true if $j \notin F$. The proof of the theorem is now complete.

Regularity hypotheses imposed on the probability matrices can be used to simplify the above results. Thus suppose that there is a value $t_{0}$ of $t$ such that $\sum_{i} p_{i j}\left(t_{0}\right)$ converges uniformly in $i$. It follows readily that $\sum_{j} p_{i j}(t)$ converges uniformly in $i$ and $t \geqq t_{0}$. This means that any limiting matrix of $P(t), t \rightarrow \infty$, has row sums 1 , so $P(t) \rightarrow U$, by Theorem 6 . A less strong condition is that there be a value $t_{0}$ of $t$, a positive integer $N$ and a positive $\epsilon$ such that $\sum_{j \leqq N} p_{i j}\left(t_{0}\right) \geqq \epsilon$ for all $i$. It follows readily that the same inequalities hold for $t \geqq t_{0}$. Then

$$
\sum_{k \leqq N} u_{j k} \geqq \epsilon ;
$$

so there can only be a finite number of $G_{\nu}$, and $U$ cannot be the null matrix. Also if $j \in F,(6.8)$ becomes

$$
\sum_{k \leqq N} \rho_{j K} u_{k} \geqq \epsilon \quad(k \in K) .
$$

Then some $\rho_{j K}>0$ for each $j \in F$, so by (6.3), $\lim _{t \rightarrow \infty} p_{i j}(t)=0$, if $i \in F, j \in F$. Thus in this case also, $P(t) \rightarrow U$, as $t \rightarrow \infty$. The fact that $P(t) \rightarrow U$ under the above hypotheses can also be derived using general theorems of Doeblin( $\left.{ }^{12}\right)$ or of Kryloff and Bogolioúboff( $\left({ }^{13}\right)$.

If there is a set of non-negative numbers $p_{1}, p_{2}, \cdots$ such that

$$
\sum_{i} p_{i} p_{i j}(t)=p_{i} \quad(\text { all } j), \quad \sum_{j} p_{j}=1,
$$

(12) Thesis, Paris, 1938, pp. 105-109.

(13) Paris, Comptes Rendus de l'Académie des Sciences, vol. 204 (1937), pp. 1454-1456. 
the set $p_{1}, \cdots$ will be called a set of (stationary) absolute probabilities. The number $p_{j}$ can be considered as the probability of being in the $j$ th state at time $t$. Any linear combination of absolute probabilities with non-negative coefficients is also a set of absolute probabilities, or proportional to a set. If $U$ is defined as in Theorem 6, the second set of equations of (6.2) states that the $i$ th row of $U$, if $i \notin F$, is a set of absolute probabilities. If $i \in F$, the $i$ th row of $U$ is a linear combination (coefficients $\rho_{i K}$ ) of the rows of elements with first subscripts not in $F$. Then every row of $U$ is a set of absolute probabilities, or proportional to a set (if the row sum is less than 1). Moreover (6.9) implies that $\sum_{i} p_{i} u_{i j}=p_{j}$; so any set of absolute probabilities is a linear combination (non-negative coefficients) of rows of $U$. The states with subscripts in $F$ then always have probability 0 , regardless of the absolute probabilities. One simple consequence of these remarks is that if there is a solution to (6.9), $U$ cannot be identically 0 , and some row of $U$ is also a solution of (6.9); there is a solution of (6.9) determined by the equations $p_{j}=\lim _{t \rightarrow \infty} p_{\alpha j}(t), \alpha$ fixed, not in $F$.

THEOREM 7. Suppose that the $p_{i j}(t)$ satisfying $(0.1)$ are continuous. Then if $U$ is defined as in Theorem 6,

$$
\lim _{T \rightarrow \infty} \frac{1}{T} \int_{0}^{T} p_{i j}(t) d t=u_{i j}
$$

for all $i, j$.

Let $Q(T)$ be the matrix with general element $q_{i j}(T)$ :

$$
q_{i j}(T)=\frac{1}{T} \int_{0}^{T} p_{i j}(t) d t
$$

Since

$$
\sum_{j} q_{i j}(T) p_{j k}(t)=\sum_{j} p_{i j}(t) q_{j k}(T)=\frac{1}{T} \int_{t}^{T+t} p_{i k}(s) d s,
$$

if $U^{\prime}$ is a limiting matrix of $Q(T), T \rightarrow \infty$, it follows that

$$
U^{\prime} P(t) \leqq P(t) U^{\prime}=U^{\prime}
$$

Since the row sums of $U^{\prime} P(t)$ are the same as those of $U^{\prime}$, there must be equality:

$$
U^{\prime} P(t)=P(t) U^{\prime}=U^{\prime}
$$

According to Theorem 6,

$$
U P(t)=P(t) U=U .
$$

It follows from (7.3) and (7.4) that 


$$
\begin{aligned}
& U U^{\prime} \leqq U^{\prime} U=U^{\prime}, \\
& U^{\prime} U \leqq U U^{\prime}=U .
\end{aligned}
$$

Then $U=U U^{\prime} \leqq U^{\prime}=U^{\prime} U \leqq U, U=U^{\prime}$, as was to be proved.

The following is a simple example illustrating the fact that $U$ in Theorems 6,7 may be the null matrix. Let $p_{i j}(t)=0$ if $j<i$, and otherwise define $p_{i j}(t)$ by

$$
p_{i j}(t)=\frac{t^{j-i}}{(j-i) !} e^{-t}
$$

Evidently $p_{i j}(t) \rightarrow 0$, as $t \rightarrow \infty$. There can be no stationary absolute probabilities in this case.

In examining the successive transitions of the system, we shall assume that the system is initially in a state $\alpha$, where $\alpha$ will be held fixed throughout the discussion. Let $\xi(t)$ be the number of the state assumed by the system at time $t$. Then $\xi(t)$, for each fixed value of $t$, is a chance variable: $\xi(0) \equiv \alpha$; $\xi(t)=j$ with probability $p_{\alpha j}(t)$ if $t>0$. To discuss the continuity properties of $\xi(t)$ in $t$ we shall assume a minimum of regularity properties of $P(t)$, to which we shall be led in a natural way. In order to discuss the probability measures under consideration, we must, as usual, find a space $\Omega^{*}$ of points $\omega$, a measure defined on $\Omega^{*}$, and a one-parameter family of measurable functions $x_{t}(\omega), 0 \leqq t<\infty$, such that the probability relations of the chance variables $\{\xi(t)\}$ become measure relations of the functions $\left\{x_{t}(\omega)\right\}$. Let $\Omega^{*}$ be the space of all functions $x(t), 0 \leqq t<\infty$, taking on the integral values used in the subscripts of $P(t)$. A probability measure on $\Omega^{*}$ is defined as follows. If $0=t_{0}<t_{1}<\cdots<t_{n}$, the conditions

$$
x\left(t_{j}\right)=\nu_{j} \quad(j=0, \cdots, n)
$$

determine a subset of $\Omega^{*}$ and the measure of this subset is defined by

$$
\begin{aligned}
& P^{*}\left\{x\left(t_{j}\right)=\nu_{j}, j=0, \cdots, n\right\} \\
& =\delta_{\alpha \nu_{0}} p_{\alpha \nu_{1}}\left(t_{1}\right) p_{\nu_{1} \nu_{2}}\left(t_{2}-t_{1}\right) \cdots p_{\nu_{n-1} \nu_{n}}\left(t_{n}-t_{n-1}\right) .
\end{aligned}
$$

By a theorem of Kolmogoroff ( $\left.{ }^{14}\right)$, a completely additive measure function is determined on $\Omega^{*}$ by these conditions. Let $x_{8}(\omega)$ be the function of $\omega: x(t)$ which takes on the numerical value $f(s)$ if $\omega$ is the function $f(t)$. Then the probability relations of the chance variables $\{\xi(t)\}$ become measure relations of the measurable functions $\left\{x_{t}(\omega)\right\}$ :

$$
P^{*}\left\{x_{t}(\omega)=j\right\}=p_{\alpha j}(t)
$$

and so forth. We shall sometimes write $x(t)$ instead of $x_{t}(\omega)$, so that " $x(t)$ " can mean, for example: (a) a point $\omega$ of $\Omega^{*}$; (b) a function $x_{t}(\omega)$ of $\omega$; (c) a num-

(14) Grundbegriffe der Wahrscheinlichkeitsrechnung. Ergebnisse der Mathematik, vol. 2, no. 4, pp. 24-30. The fact that our functions assume only integral values, whereas those of Kolmogoroff assume all values necessitates only trivial changes in the proof. 
ber, the value of the function $x(t)$ at the point $t$. When there is any danger of confusion, the proper meaning will be explicitly stated. The function $x_{t}(\omega)$ is automatically defined on any subset of $\Omega^{*}$, and it is usually desirable to restrict $\omega$ to be in a subset $\Omega$ of $\Omega^{*}$, of outer measure 1 , defining a $P$-measure on $\Omega$ by setting $P\left(\Lambda^{*} \cdot \Omega\right)=P^{*}\left(\Lambda^{*}\right)$ for any $P^{*}$-measurable set $\left.{ }^{15}\right) \Lambda^{*}$. The probability relations of the chance variables $\{\xi(t)\}$ now become the measure relations of the functions $x_{t}(\omega), \omega \in \Omega: P\left\{x_{s}(\omega)=j\right\}=p_{\alpha j}(s)$ and so on $\left({ }^{16}\right)$. It has been shown $\left({ }^{17}\right)$ that if any $P^{*}$-measure is given, there always corresponds an everywhere dense denumerable sequence of real numbers $R:\left\{r_{j}\right\}$ such that if $I$ is any open interval, and if $s \in I$,

$$
P^{*}\left\{\underset{r_{j} \in I}{\text { G.L.B. }} x\left(r_{j}\right) \leqq x(s) \leqq \underset{r_{j} \in I}{\text { L.U.B. }} x\left(r_{j}\right)\right\}=1\left({ }^{18}\right) .
$$

It has been shown $\left({ }^{19}\right)$ that $\Omega$ can be chosen to consist of all (possibly infinitevalued) functions $x(t)$ which satisfy the relation

$$
\lim _{r_{j} \rightarrow t} x\left(r_{j}\right) \leqq x(t) \leqq \limsup _{r_{j} \rightarrow t} x\left(r_{j}\right)
$$

for all $t \in R$. Then if this is done,

$$
\underset{r_{j} \in I}{\text { G.L.B. }} x\left(r_{j}\right) \leqq x(s) \leqq \underset{r_{j} \in I}{\text { L.U.B. }} x\left(r_{j}\right)
$$

for all $s \in I, \omega: x(t)$ in $\Omega$, sharpening (8.3). Such a space $\Omega$ is called quasiseparable, and the process : that is, the combination of $\Omega$ with its $P$-measure, is called a quasi-separable process.

A measure can be defined on the space $T \times \Omega$ of couples $(t, \omega)$, as the product of Lebesgue measure on the $t$-axis and $P$-measure on $\omega$. The process is called measurable if the function $x_{t}(\omega)$ is $(t, \omega)$-measurable. The $P^{*}$-measure is then said to determine a measurable process. This hypothesis on the $P^{*}$-measure is certainly a minimum hypothesis. On the other hand, there are natural analytic restrictions on the $p_{i j}(t)$. Let $G_{\alpha}$ be the set of subscripts $j$ such that $p_{\alpha j}(t) \not \equiv 0$. Only the subscripts in $G_{\alpha}$ need be considered in analyzing the transitions of the system, supposed initially in state $\alpha$. It follows readily from (0.1) that $p_{i j}(t) \equiv 0$ if $i \in G_{\alpha}, j \notin G_{\alpha}$. The matrix $P_{\alpha}(t): p_{i j}(t)$ with $i, j \in G_{\alpha}$ then satisfies $(0.1)$, and it is this matrix $P_{\alpha}(t)$ which is essential to the discussion. The

(15) Cf. Doob, these Transactions, vol. 42 (1937), pp. 108-110.

(16) $P\left\{x_{s}(\omega)=j\right\}$ is to be interpreted as the $\Omega$-measure of the set of all functions $x(t)$ in $\Omega$ for which $x(s)=j$.

(17) Doob, these Transactions, vol. 47 (1940), p. 467.

(18) This equality holds for each fixed $s$. The $\omega$-set $\{x(s) \leqq \phi, s \in I\}, \phi$ a $P^{*}$-measurable function, is not $P^{*}$-measurable, so each value of $s$ must be considered separately in (8.3), or in probability relations of similar type. The subspace $\Omega$ is introduced in order to avoid this necessity.

(19) Op. cit., pp. 468-469. 
natural analytic hypotheses on $P_{\alpha}(t)$ would include the measurability of its elements. This, by Theorem 1 , implies their continuity, and then (Theorem 4), $\lim _{t \rightarrow 0} P_{\alpha}(t)=U$ exists. The matrix $U$ is the first determining factor of the regularity of the process. It is natural to suppose that it is the identity matrix. A glance at Theorem 4 shows that no other hypothesis can possibly be compatible with any sort of continuity in the transitions of the system.

These considerations lead to the following formulation of a natural hypothesis to be imposed on the matrix function $P_{\alpha}(t)$. We shall denote as hypothesis $H_{\alpha}$ the hypothesis that the system is initially in state $\alpha$, and that if $i \in G_{\alpha}, \lim _{t \rightarrow 0} p_{i i}(t)=1$. Then $\lim _{t \rightarrow 0} p_{i j}(t)=\delta_{i j}\left(i \in G_{\alpha}\right)$. Since $P_{\alpha}(t)$ satisfies $(0.1)$, the $p_{i j}(t)\left(i, j \in G_{\alpha}\right)$ will be continuous (Theorem 4$)$. Moreover $p_{i j}(t) \equiv 0$ if $i \in G_{\alpha}, j \notin G_{\alpha}$. Then if hypothesis $H_{\alpha}$ is true, and if $i \in G_{\alpha}, p_{i j}(t)$ is continuous for all $j$, and $\lim _{t \rightarrow 0} p_{i j}(t)=\delta_{i j}$. Hypothesis $H_{\alpha}$ implies the continuity of $p_{\alpha j}(t)$ for all $j$, even though $\alpha$ may not be in $G_{\alpha}$. In fact the equation

$$
p_{\alpha j}(s+h)=\sum_{i \in G \alpha} p_{\alpha i}(s) p_{i j}(h)
$$

shows that $p_{\alpha j}(t)$ is continuous for $t>s$, and therefore for all $t$. If $i \in G_{\alpha}$, then $p_{i i}(t)>0$ for all $t$, and if $i=\alpha, j \in G_{\alpha}$ or if $i, j \in G_{\alpha}$ then $p_{i j}(t)=0$ at most on a finite interval $0<t \leqq t_{0}$ (depending on $i, j$ ). The first fact follows from the inequality $p_{i i}(t) \geqq p_{i i}(t / n)^{n}, n=1,2, \cdots$, since $\lim _{n \rightarrow \infty} p_{i i}(t / n)=1$. The second fact follows from the inequality $p_{i j}(t+h) \geqq p_{i j}(t) p_{i j}(h)$ which implies that if $p_{i j}\left(t^{\prime}\right)>0$, then $p_{i j}(t)>0$, for $t>t^{\prime}$.

The following theorem shows the relations between various hypotheses it would be natural to assume.

Theorem 8. Suppose that $P^{*}\{x(0)=\alpha\}=1$. Then the following three conditions on $P^{*}$-measure are equivalent.

(i) The $P^{*}$-measure determines a measurable process.

(ii) Hypothesis $H_{\alpha}$ is satisfied.

(iii) For every $\tau>0$,

$$
\lim _{t \rightarrow \tau} P^{*}\{x(t)=x(\tau)\}=1 .
$$

In the usual language of measure theory, (8.6) states that $x_{t}(\omega) \rightarrow x_{\tau}(\omega)$ in measure. We shall prove a much stronger result below, Theorem 11 . To prove Theorem 8, we prove that (i) implies (ii), (ii) implies (iii), and (iii) implies (i).

Proof that (i) implies (ii). Suppose that $P^{*}$-measure determines a measurable process. Then it follows $\left({ }^{20}\right)$ that for fixed $h>0, \epsilon>0, P^{*}\{|x(t+h)-x(t)|$ $>\epsilon\}$ is Lebesgue measurable in $t$, and (as $h \rightarrow 0$ ) goes to 0 in measure on any finite $t$-interval. If $\epsilon<1$,

$$
P^{*}\{|x(t+h)-x(t)|>\epsilon\}=\sum_{j \in G_{\alpha}} p_{\alpha j}(t)\left[1-p_{i j}(h)\right] .
$$

(20) Doob, these Transactions, vol. 42 (1937), p. 117. 
Since the quantity in (8.7) goes to 0 as $h \rightarrow 0$ in measure on every finite $t$-interval, and since $p_{\alpha j}(t)>0$ if $t$ is sufficiently large, $\lim _{h \rightarrow 0} p_{j j}(h)=1$ if $j \in G_{\alpha}$. Then hypothesis $H_{\alpha}$ is satisfied.

Proof that (ii) implies (iii). If hypothesis $H_{\alpha}$ is true, we shall prove (8.6) by evaluating the probabilities involved. If $0<\tau<t$,

$$
\begin{aligned}
P^{*}\{x(t)=x(\tau)\} & =\sum_{j} P^{*}\{x(t)=x(\tau)=j\} \\
& =\sum_{j \in G_{\alpha}} p_{\alpha j}(\tau) p_{j j}(t-\tau)
\end{aligned}
$$

and if $0<t<\tau$,

$$
P^{*}\{x(t)=x(\tau)\}=\sum_{j \in G_{\alpha}} p_{\alpha j}(t) p_{j j}(\tau-t) .
$$

Proof that (iii) implies (i). Condition (iii) is known (loc. cit. $\left({ }^{20}\right)$ ) to imply that the $P^{*}$-measure determines a measurable process.

Now the series in (8.8) is majorized by $\sum_{j} p_{\alpha j}(\tau)$, and that in $\left(8.8^{\prime \prime}\right)$ by $\sum_{j} p_{\alpha j}(t)$. Then the series in (8.8) converges uniformly in $t$. The series $\sum_{j} p_{\alpha j}(t)$ is a series of non-negative continuous functions, converging to the continuous function 1 , so there is uniform convergence in a neighborhood of $\tau$. Thus the series in (8.8) and $\left(8.8^{\prime}\right)$ are uniformly convergent for $t$ near $\tau$, and when $t \rightarrow \tau$ both become $\sum_{j} p_{\alpha j}(\tau)=1$, as was to be proved.

THEOREM 9. Suppose that hypothesis $H_{\alpha}$ is true. Then if $i \in G_{\alpha}$,

$$
\lim _{t \rightarrow 0} \frac{1-p_{i i}(t)}{t}=q_{i}(\leqq+\infty)
$$

exists. If $q_{i}=0, p_{i i}(t) \equiv 1$. If $q_{i}=\infty$, then if $j \neq i, j \in G_{\alpha}$,

$$
\lim _{t \rightarrow 0} \frac{p_{i j}(t)}{1-p_{i i}(t)}=\lim _{t \rightarrow 0} \frac{p_{j i}(t)}{1-p_{i i}(t)}=0 .
$$

If $q_{i}<\infty$, then for $j \neq i, j \in G_{\alpha}$ the limits

$$
\lim _{t \rightarrow 0} \frac{p_{i j}(t)}{t}=q_{i j}(<+\infty), \quad \lim _{t \rightarrow 0} \frac{p_{i i}(t)}{t}=q_{j i}(<+\infty)
$$

exist, and

$$
\sum_{j} q_{i j} \leqq q_{i}
$$

In the finite-dimensional case ${ }^{(21)}, q_{i}<\infty$ for all $i \in G_{\alpha}$, and there is equality in (9.4).

Let $R$ be a denumerable everywhere dense $t$-set. Since when $t \rightarrow \tau$, (21) Doeblin (I) proved Theorem 9 in the finite-dimensional case. 
$x(t) \rightarrow x(\tau)$ in measure (Theorem 8 ), it follows that

$$
\liminf _{r \rightarrow \tau} x(r) \leqq x(\tau) \leqq \limsup _{r \rightarrow \tau} x(r)
$$

with probability 1 (that is, almost everywhere on $\Omega^{*}$ ). Then $(8.3)$ is satisfied. We shall also need the following fact: If $I$ is any open $t$-interval, and if $t_{1}^{(n)}<\cdots<t_{\nu_{n}}^{(n)}$ are points in $I$, with $\max _{j}\left(t_{j}^{(n)}-t_{j-1}^{(n)}\right)=\delta_{n}$, then if $\delta_{n} \rightarrow 0$,

$$
\lim _{n \rightarrow \infty} \text { L.U.B. } x\left(t_{j}^{(n)}\right)=\underset{r \in R \cdot I}{\text { L.U.B. }} x(r)
$$

with probability 1 . This can be proved as follows. Because of the fact that when $t \rightarrow \tau, x(t) \rightarrow x(\tau)$ in measure, it surely is true that for each $r$ in $I$,

$$
\underset{n \rightarrow \infty}{\lim \inf \text { L.U.B. }} x\left(t_{j}^{(n)}\right) \geqq x(r)
$$

with probability 1 , and (9.7) implies (9.6), because of (8.3). In the same way we can prove

$$
\lim _{n \rightarrow \infty} \text { G.L.B. } x\left(t_{j}^{(n)}\right)=\underset{r \in R \cdot I}{\text { G.L.B. }} x(r)
$$

with probability 1.

Now let $i \in G_{\alpha}$, and choose $\tau$ so that $p_{\alpha i}(\tau)>0$. Let $\phi_{i}(h)$ be the probability that if $x(\tau)=i$ then $x(r)=i$ for $\tau \leqq r \leqq \tau+h(r \in R)$. (If $R$ is used to determine a quasi-separable process, $\phi_{i}(h)$ is the probability that if $x(\tau)=i$, then $x(t)=i$ for $\tau \leqq t \leqq \tau+h$.) According to (9.6) and (9.8), if $\tau=t_{1}^{(n)}<\cdots \leqq h$, and $\max _{j}\left(t_{j}^{(n)}-t_{j-1}^{(n)}\right)=\delta_{n} \rightarrow 0$, then

$$
\begin{aligned}
P^{*}\{x(r) & =i, \tau \leqq r \leqq \tau+h,(r \in R)\}=\lim _{n \rightarrow \infty} P^{*}\left\{x\left(t_{j}^{(n)}\right)=i, j \geqq 1\right\} \\
& =p_{\alpha i}(\tau) \lim _{n \rightarrow \infty} \prod_{j \geqq 1} p_{i i}\left(t_{j+1}^{(n)}-t_{j}^{(n)}\right)=p_{\alpha i}(\tau) \phi_{i}(h) .
\end{aligned}
$$

Let $\left\{\epsilon_{n}\right\}$ be any sequence of positive numbers converging to 0 . To prove (9.1) it will be sufficient to let $t \rightarrow 0$ through the sequence $\left\{\epsilon_{n}\right\}$, and to show that there is a limit, which is independent of the sequence $\left\{\epsilon_{n}\right\}$. Choose the integers $m_{n}$ so that $m_{n} \epsilon_{n} \uparrow h$. Then setting $t_{j+1}^{(n)}-\tau=j \epsilon_{n}$ in (9.9), $0 \leqq j \leqq m_{n}$,

$$
\lim _{n \rightarrow \infty} p_{i i}\left(\epsilon_{n}\right)^{m_{n}}=\phi_{i}(h) \text {. }
$$

This implies that if $\phi_{i}(h)>0$

$$
\lim _{n \rightarrow \infty} m_{n} \log p_{i i}\left(\epsilon_{n}\right)=-\lim _{n \rightarrow \infty} h \frac{1-p_{i i}\left(\epsilon_{n}\right)}{\epsilon_{n}}=\log \phi_{i}(h) .
$$

We have thus shown that unless $\phi_{i}(h)=0,(9.1)$ is true, and 


$$
\phi_{i}(h)=e^{-q_{i} h} .
$$

On the other hand, if $\phi_{i}(h)=0,(9.1)$ is true with $q_{i}=\infty$, and then $\phi_{i}(h) \equiv 0$. Since $p_{i i}(t) \geqq \phi_{i}(t), q_{i}=0$ implies that $p_{i i}(t) \equiv 1$. In proving (9.2) and (9.3) we can assume that $p_{i i}(t)<1$ for all $t$, since otherwise $(0.1)$ implies that $p_{i i}(t) \equiv 1$, so $q_{i}=0:$ in this case $(9.2)$ is inapplicable; the first part of $(9.3)$ is obvious, and the second is proved by a trivial modification of the proof below. To prove (9.2) we note that if $\eta>0, j \neq i, j \in G_{\alpha}$,

$$
p_{i j}(n \epsilon) \geqq \sum_{k=0}^{n-1} p_{i i}(\epsilon)^{k} p_{i j}(\epsilon) p_{j j}(\overline{n-k-1} \epsilon) \geqq(1-\eta) \frac{1-p_{i i}(\epsilon)^{n}}{1-p_{i i}(\epsilon)} p_{i j}(\epsilon),
$$

if $n \epsilon$ is sufficiently small. Then if $q_{i}=\infty$, when $n \rightarrow \infty$ and $\epsilon \rightarrow 0$ so that $n \epsilon^{-\rightarrow}$, (9.13) becomes

$$
p_{i j}(t) \geqq(1-\eta) \limsup _{\epsilon \rightarrow 0} \frac{p_{i j}(\epsilon)}{1-p_{i i}(\epsilon)},
$$

for sufficiently small $t$. When $t \rightarrow 0$ this gives the first part of (9.2). Similarly if $i \neq j, j \in G_{\alpha}$

$$
p_{j i}(n \epsilon) \geqq \sum_{k=0}^{n-1} p_{j i}(\overline{n-k-1} \epsilon) p_{j i}(\epsilon) p_{i i}(\epsilon)^{k} \geqq(1-\eta) \frac{1-p_{i i}(\epsilon)^{n}}{1-p_{i i}(\epsilon)} p_{j i}(\epsilon)
$$

is true for sufficiently small $n$, and then if $q_{i}=\infty$

$$
p_{j i}(t) \geqq(1-\eta) \limsup _{\epsilon \rightarrow 0} \frac{p_{j i}(\epsilon)}{1-p_{i i}(\epsilon)},
$$

for sufficiently small $t$. When $t \rightarrow 0$ this gives the second part of (9.2). If $q_{i}<\infty$, (9.13) implies that

$$
p_{i j}(t) \geqq(1-\eta) \frac{1-e^{-q_{i} t}}{q_{i}} \limsup _{\epsilon \rightarrow 0} \frac{p_{i j}(\epsilon)}{\epsilon} .
$$

Then

$$
\liminf _{t \rightarrow 0} \frac{p_{i j}(t)}{t} \geqq(1-\eta) \limsup _{\epsilon \rightarrow 0} \frac{p_{i j}(\epsilon)}{\epsilon} .
$$

Since $\eta>0$ is arbitrary, this implies that $\lim _{t \rightarrow 0} p_{i j}(t) / t$ exists, and the limit is finite, from (9.15). Similarly equation $\left(9.13^{\prime}\right)$ implies that $\lim _{t \rightarrow 0} p_{j i}(t) / t$ exists and is finite. Moreover

$$
\sum_{j \neq i} \frac{p_{i j}(t)}{t}=\frac{1-p_{i i}(t)}{t},
$$

so that (9.4) is true. Equation (9.17) can also be written 


$$
\sum_{j \neq i} \frac{p_{i j}(t)}{1-p_{i i}(t)}=1 .
$$

Then in the finite-dimensional case $q_{i}=\infty$ is impossible (since each term of the sum goes to 0 with $t$ if $\left.q_{i}=\infty\right)$, and (9.17) implies that there is equality in (9.4).

In discussing the continuity properties of $x(t)$ in $t$, it is usually convenient, because of measurability considerations, to choose a denumerable everywhere dense $t$-set $R$ and then consider the functions $x(r)$ for $r \in R$. The continuity properties of $x(r)$ can be interpreted as continuity properties of $x(t)$, if the proper space $\Omega$ of the stochastic process is chosen, and this will sometimes be done below.

Theorem 10. Suppose that hypothesis $H_{\alpha}$ is true. Let $\tau$ be any positive number and let $R$ be any denumerable everywhere dense set. Then $\lim _{r \rightarrow \tau} x(r)=x(\tau)$ $(r \in R)$ with probability 1 if and only if whenever $p_{\alpha i}(\tau)>0, q_{i}$ is finite. If $q_{i}<\infty$ and if $p_{\alpha i}(\tau)>0, q_{i j} / q_{i}$ is the conditional probability that if $x(\tau)=i$, and if there is a discontinuity of $x(r)(r \in R)$ before $\tau+h$, then there is a first discontinuity before $\tau+h$, which is an isolated discontinuity where $x(r)$ jumps to $j$.

The probability that $x(\tau)=i$ and that $x(r)=i$ for $r \in R, \tau-h<r<\tau+h$ is $p_{\alpha i}(\tau-h) \phi_{i}(2 h)$. Then $\lim _{r \rightarrow \tau} x(r)=x(\tau)$ with probability 1 if and only if $\lim _{h \rightarrow 0} \phi_{i}(h)=1$, that is, if and only if $q_{i}<\infty$, whenever $p_{\alpha i}(\tau)>0$. This proves the first part of the theorem. The second part requires a more detailed analysis. Suppose that $q_{i}<\infty$ and that $p_{\alpha i}(\tau)>0$. We shall evaluate the probability of the $x(t)$-set $\Lambda_{\eta}$ determined by the following conditions: $x(\tau)=i ; x(r)=i$ for $r \in R, r>\tau$ on some interval of $r$-values; $x(r)$ then jumps to $j$, remaining equal to $j$ on some interval of length at least $\eta$, the jump occurring before $\tau+h$. Let $n$ be any positive integer, and define $\Lambda_{n, \eta}$ by

$$
\begin{aligned}
\Lambda_{n, \eta}=\sum_{m=1}^{n-2}\{x(\tau)=i ; x(r) & \equiv i, \tau<r<\tau+\frac{m}{n} h ; x\left(\tau+\frac{m+1}{n} h\right)=j ; \\
x(r) & \left.\equiv j, \tau+\frac{m+1}{n} h<r<\tau+\frac{m+1}{n} h+\eta\right\} .
\end{aligned}
$$

Then

$$
\begin{aligned}
P\left(\Lambda_{n, \eta}\right) & =\sum_{m=1}^{n-2} p_{\alpha i}(\tau) e^{-m h q_{i} / n} p_{i j}(h / n) e^{-\eta q_{j}} \\
& =p_{\alpha i}(\tau) \frac{e^{-h q_{i} / n}-e^{-h q_{i}(1-1 / n)}}{1-e^{-h q_{i} / n}} p_{i j}(h / n) e^{-\eta q_{j}} \\
& \rightarrow p_{\alpha i}(\tau) \frac{1-e^{-h q_{i}}}{q_{i}} q_{i j} e^{-\eta q_{j}} .
\end{aligned}
$$


Now if $x(t) \in \Lambda_{\eta}$ it follows that $x(t) \in \Lambda_{n, \eta^{\prime}}$ for sufficiently large $n$, whenever $\eta^{\prime}<\eta$ :

$$
\Lambda_{\eta} \subset \liminf _{n \rightarrow \infty} \Lambda_{n, \eta^{\prime}}
$$

If $x(t) \in \Lambda_{n, \eta}$ for infinitely many values of $n, x(t) \in \Lambda_{\eta}$, if no $\tau+(m / n) h \in R$ :

$$
\limsup _{n \rightarrow \infty} \Lambda_{n, \eta} \subset \Lambda_{\eta} \text {. }
$$

Then if $\eta^{\prime}<\eta$

$$
\limsup _{n \rightarrow \infty} P\left(\Lambda_{n, \eta}\right) \leqq P\left(\Lambda_{\eta}\right) \leqq \liminf _{n \rightarrow \infty} P\left(\Lambda_{n, \eta^{\prime}}\right) .
$$

The inferior and superior limits in (10.5) are actual limits, evaluated in (10.2). Since the limit function of $\eta$ is continuous, we obtain $\left({ }^{22}\right)$, letting $\eta^{\prime} \rightarrow \eta$,

$$
P\left(\Lambda_{\eta}\right)=p_{\alpha i}(\tau)\left(1-e^{-h q_{i}}\right) \frac{q_{i j}}{q_{i}} e^{-\eta q_{j}} .
$$

The probability that $x(\tau)=i$, that $x(r)=i$ for $r>\tau$ on some $r$-interval $(r \in R)$, and that then $x(r)$ jumps to $j$ where it remains for some $r$-interval, the jump occurring before $\tau+h$, is therefore

$$
\lim _{\eta \rightarrow 0} P\left(\Lambda_{\eta}\right)=p_{\alpha i}(\tau)\left(1-e^{-h q_{i}}\right) q_{i j} / q_{i},
$$

and this equality is equivalent to the statement of the theorem.

To make clear the meaning of Theorem 10 , suppose that $\sum_{j} q_{i j}=q_{i}<\infty$ for all $i$ in $G_{\alpha}$. Then if $\tau>0, \lim _{r \rightarrow \tau} x(r)=x(\tau)$ with probability 1 . Excluding an $x(t)$-set of $\Omega^{*}$-measure 0 , each $x(t)$ in the remainder $\Lambda$ is then equal on $R$ to $x(\tau)$ for $r$ sufficiently near $\tau$. According to the second part of the theorem, we can make the excluded $\Omega^{*}$-set so large that if $x(t) \in \Lambda$ there will be a first discontinuity of $x(r)$ (if any) after $\tau$, a jump. Now, applying the second part of the theorem, letting $\tau$ run through all rational numbers, we see that the excluded $\Omega^{*}$-set can be made so large that if $x(t) \in \Lambda$, there will be a second discontinuity (if there is more than one), also a jump, a third, and so on. These discontinuities may cluster at a point, to give $x(r)$ a discontinuity which is no longer a jump.

We shall use a somewhat indirect method in examining in more detail the transitions of the system, that is, the discontinuities of $x(t)$. This method has the advantage of exhibiting analytically the relation between the regularity of the matrix function $\left(p_{i j}(t)\right)$ and the discontinuities of $x(t)$.

(22) We have tacitly assumed the measurability of $\Lambda_{\eta}$. This is easily proved directly, or the above discussion can be modified, using inner and outer measures in (10.5), to furnish the proof that $\Lambda_{\eta}$ is measurable, besides evaluating its measure. The restriction we have made on $h$ is essential for (10.4), but evidently (10.5) and (10.6) are true without this restriction. 
Let $y_{t}$ (for each $t$ in some point set) be a chance variable. The family of chance variables $\left\{y_{t}\right\}$ will be said to have the property $\varepsilon$ if (for any natural number $n$ ), whenever $t_{1}<\cdots<t_{n+1}$,

$$
E\left\{y_{t_{1}}, \cdots, y_{t_{n}} ; y_{t_{n+1}}\right\}=y_{t_{n}}\left({ }^{23}\right),
$$

with probability $1\left({ }^{24}\right)$. Suppose a family $\left\{y_{t}\right\}$ has the property $\mathcal{E}$, for $t$ in some interval $(a, b)$. Then if $a<\tau<b, t_{n} \uparrow \tau$ implies that $\lim _{n \rightarrow \infty} y_{t_{n}}=y_{\tau-}$ exists with probability 1 , and the limit $y_{\tau-}$ is independent (neglecting zero probabilities) of the particular sequence $\left\{t_{n}\right\}$. The chance variable $\left\{y_{\tau_{+}}\right\}$is defined similarly in terms of approach from above. Moreover, $y_{\tau-}=y_{\tau+}=y_{\tau}$ with probability 1 , if $\tau$ is not in some set, which is at most denumerable. We shall call this set the set of fixed discontinuities. If $R$ is any denumerable set, dense on $(a, b)$, $y_{r}(r \in R)$, with probability 1 , considered as a function of $r$ alone is equal to a function defined on $(a, b)$, and continuous on the right at every point of $(a, b)$ not a fixed discontinuity point. It will be useful below to say that a family of chance variables $y_{t}$ has the property $\varepsilon^{*}$ if the family $y_{-t}$ has the property $\mathcal{E}$.

It is easily verified that if $T>0$, and if $y_{t}$ is defined by

$$
y_{t}=p_{x(t) j}(T-t)
$$$$
(0<t<T),
$$

then the family of chance variables has the property $\mathcal{E}\left({ }^{25}\right)$. We shall show that there are no fixed discontinuities if hypothesis $H_{\alpha}$ is true. To do this it will be sufficient to show that if $t$ is given, and if $t_{n} \rightarrow t$, then some subsequence of $\left\{y_{t_{n}}\right\}$ converges to $y_{t}$ with probability 1 . Since according to Theorem 8 , $x_{t_{n}}(\omega)$ converges to $x_{t}(\omega)$ in measure, some subsequence, $x_{\tau_{n}}(\omega)$, converges to $x_{t}(\omega)$ with probability 1 . Then

$$
y_{\tau_{n}}=p_{x(t) j}\left(T-\tau_{n}\right)
$$

for large $n$, with probability 1 , so that $y_{\tau_{n}} \rightarrow y_{t}$ with probability 1 , because the $p_{i j}(t)$ are continuous if $i \in G_{\alpha}$ and for each $t, P^{*}\left\{x_{t}(\omega) \in G_{\alpha}\right\}=1$. In a similar way it can be proved that if $T^{*}>0$, and if $y_{t}^{*}$ is defined by

$$
y_{t}^{*}=\frac{p_{j x(t)}\left(t-T^{*}\right)}{p_{\alpha x(t)}(t)}
$$

the family of chance variables $\left\{y_{t}^{*}\right\}$ has the property $\mathcal{E}^{*}$, and there are no fixed discontinuities, if hypothesis $H_{\alpha}$ is true. The chance variable $y_{t}^{*} \cdot p_{\alpha j}\left(T^{*}\right)$

(23) The notation $E\left\{y_{t_{1}}, \cdots, y_{t_{n}} ; y_{t_{n+1}}\right\}$ will be used to denote the conditional expectation of $y_{t_{n+1}}$ for given values of $y_{t_{1}}, \cdots, y_{t_{n}}$, a function of the latter variables.

${ }^{24}$ ) The properties of such a family, summarized here, are proved in the author's paper in these Transactions, vol. 47 (1940), pp. 455-486. This paper will be referred to as " $\varepsilon$."

(25) This fact is a result of the well known relations between conditional expectation functions, and is a special case of the fact that if $\left\{w_{t}\right\}$ is any family of chance variables, if $z$ is a chance variable dependent on the $w_{t}$, and if $z_{t}=E\left\{w_{s}, s \leqq t ; z\right\},\left(z_{t}=\right.$ expectation of $z$ for $w_{s}$ given for $s \leqq t$ ), then the family $\left\{z_{t}\right\}$ has the property $\varepsilon$. 
bears the same relation to the inverse process ( $t$ decreasing) as $y_{t}$ bears to the given process. For each $t$, the denominator in (11.4) vanishes only with probability 0 (hypothesis $H_{\alpha}$ ). The following two regularity conditions on the $p_{i j}(t)$ will be useful.

Condition $C(\beta)$. Let $\beta$ be in $G_{\alpha}$. Then there are numbers $\eta, \delta$ such that for all $i \neq \beta$ in $G_{\alpha}$, and all $s<\delta$

$$
p_{i \beta}(s)<1-\eta .
$$

Condition $C^{*}(\beta, \tau)$. Let $\beta$ be in $G_{\alpha}$ and let $\tau$ be a positive number with $p_{\alpha \beta}(\tau)>0$. There are positive numbers $\eta, \delta$ such that if $0<s<\delta, i \notin \beta, p_{\alpha i}(\tau)>0$, then

$$
p_{\beta i}(s)<\frac{p_{\alpha i}(\tau+s)}{p_{\alpha \beta}(\tau)}(1-\eta) .
$$

Under hypothesis $H_{\alpha}$, if $i$ is fixed and $\delta \rightarrow 0$ in (11.5), the inequality becomes $0 \leqq 1-\eta$, and under the same circumstances, $\left(11.5^{*}\right)$ becomes

$$
0 \leqq \frac{p_{\alpha i}(\tau)}{p_{\alpha \beta}(\tau)}(1-\eta)
$$

Then conditions $C(\beta)$ and $C^{*}(\beta, \tau)$ are certainly always satisfied in the finitedimensional case, under hypothesis $H_{\alpha}$, for all possible $\beta$ and pairs $\beta, \tau$ $\left(\beta \in G_{\alpha}\right)$, respectively.

Condition $C(\beta)$ can be put in an interesting alternate form. If condition $C(\beta)$ is not satisfied, there is a sequence of distinct integers $\left\{i_{\nu}\right\}$ in $G_{\alpha}$, and a sequence $\left\{s_{\nu}\right\}, s_{\nu} \rightarrow 0$, such that $p_{i_{\nu} \beta}\left(s_{\nu}\right) \rightarrow 1$. Now if $t>0$, and if $\nu$ is so large that $s_{\nu}<t$,

$$
p_{i_{\nu} j}(t)=p_{i_{\nu} \beta}\left(s_{\nu}\right) p_{\beta j}\left(t-s_{\nu}\right)+\sum_{k \neq \beta} p_{i_{\nu} k}\left(s_{\nu}\right) p_{k j}\left(t-s_{\nu}\right) .
$$

If $j \notin G_{\alpha}, p_{i_{\nu}}(t) \equiv 0$. If $j \in G_{\alpha}$, the sum on the right is at most

$$
\sum_{k \neq \beta} p_{i_{\nu} k}\left(s_{\nu}\right)=1-p_{i_{\nu} \beta}\left(s_{\nu}\right) \rightarrow 0 .
$$

Then (11.7) implies

$$
\lim _{\nu \rightarrow \infty} p_{i_{\nu} j}(t)=p_{\beta j}(t),
$$

for all $j, t$. Thus (under hypothesis $H_{\alpha}$ ) condition $C(\beta)$ is satisfied if (and, as is easily seen, only if) no sequence of distinct rows (whose elements have first subscripts in $G_{\alpha}$ ) converges, element by element to the $\beta$ th row, for all $t$. An analogous but less elegant form of $C^{*}(\beta, \tau)$ can be obtained.

The following theorem makes Theorem 10 more precise. 
THeOREM 11. Suppose that hypothesis $H_{\alpha}$ is true. Let $\tau$ be any positive number, and suppose that $R$ is any denumerable set having $\tau$ as a limit point. Then

$$
\lim _{r \rightarrow \tau} \frac{x(r)-x(\tau)}{1+x(r)^{2}}=0
$$

with probability 1. If $p_{\alpha \beta}(\tau)>0$, then $\lim _{r \rightarrow \tau} x(r)=\beta$ whenever $x(\tau)=\beta$, (with probability 1) if and only if $q_{\beta}<\infty$. If $C(\beta)$ or $C^{*}(\beta, \tau)$ is satisfied, then $q_{\beta}<\infty$.

Since, as we have seen in Theorem 8 , if $r \rightarrow \tau, x_{r}(\omega) \rightarrow x_{\tau}(\omega)$ in measure, that is, $x(r) \rightarrow x(\tau)$ in measure, it is impossible that $|x(r)| \rightarrow \infty$ with positive probability along any sequence of $r$-values approaching $\tau$. Therefore, neglecting 0 probabilities ( $\Omega^{*}$-sets of measure 0$),(11.9)$ implies that $x(r)$ always has $x(\tau)$ as a limiting value when $r \rightarrow \tau$, (the only finite limiting value) but $x(r)$ may also have $\pm \infty$ as a limiting value. In the course of the proof of Theorem 10, we have already proved that if $p_{\alpha \beta}(\tau)>0, \lim _{r \rightarrow \tau} x(r)=\beta$ whenever $x(\tau)=\beta$ with probability 1 , if and only if $q_{\beta}<\infty$ : in fact this statement follows directly from our evaluation of $\phi_{\beta}(h)$. To prove (11.9) we shall use the families of chance variables $\left\{y_{t}\right\},\left\{y_{t}^{*}\right\}$ introduced above. Since these families have no fixed discontinuities,

$$
\lim _{r \rightarrow \tau} p_{x(r) j}(T-r)=p_{x(\tau) j}(T-\tau),
$$

with probability 1 . Let $\Lambda$ be an $\omega$-set of measure 1 , such that the following conditions are satisfied, if $x(t) \in \Lambda$ :

(a) (11.10) is true for all $j$ and rational $T>\tau$;

(b) if $p_{\alpha j}(r)=0$, then $x(r) \neq j$; if $p_{\alpha j}(\tau)=0$, then $x(\tau) \neq j$.

Now suppose that $x_{0}(t) \in \Lambda$, and suppose that $x_{0}(\tau)=\beta$. Suppose that $\lim \inf _{r \rightarrow \tau}\left|x_{0}(r)\right|<+\infty$. Then there is an integer $\gamma$ such that $x_{0}(r)=\gamma$ for infinitely many values of $r$, as $r \rightarrow \tau$. Condition (a) implies

$$
\lim _{r \rightarrow \tau} p_{\gamma j}(T-r)=p_{\gamma j}(T-\tau)=p_{\beta j}(T-\tau),
$$

for all $j$ and rational $T>\tau$. (We are using the fact that because of condition (b), $p_{\alpha \gamma}(t) \not \equiv 0$, so, in accordance with hypothesis $H_{\alpha}, p_{\alpha \gamma}(t)$ is continuous in $t$.) Because of hypothesis $H_{\alpha}, \lim _{t \rightarrow 0} p_{\gamma j}(t)=\delta_{\gamma j}, \lim _{t \rightarrow 0} p_{\alpha j}(t)=\delta_{\alpha j}$ for all $j$. Thus if we let $T \rightarrow \tau$, (11.11) implies that $\delta_{\gamma j}=\delta_{\beta j}$ for all $j: \gamma=\beta$. We have now proved that the only possible limiting values of $x_{0}(r)$ as $r \rightarrow \tau$ are $x_{0}(\tau), \pm \infty$; (11.9) is true for $x(t) \in \Lambda$, and hence is true with probability 1 . If the matrices are finite-dimensional, there can be only finite limiting values of $x_{0}(r)$; so $x(r) \rightarrow x(\tau)(r \in R)$ with probability 1 , as we have already proved in Theorem 10. Now suppose that $\left(p_{i j}(t)\right)$ is infinite-dimensional, and suppose that for some $x_{0}(t)$ in $\Lambda, x_{0}(r)$ does not approach $x_{0}(\tau)=\beta(r \in R)$. Then there must be a sequence of integers $\left\{i_{\nu}\right\}$ and a sequence $\left\{r_{\nu}\right\}$ such that $x_{0}\left(r_{\nu}\right)=i_{\nu} \rightarrow \pm \infty$, $\left(r_{\nu} \rightarrow \tau\right)$. Then (11.10) becomes 


$$
\lim _{\nu \rightarrow \infty} p_{i_{\nu} j}\left(T-r_{\nu}\right)=p_{\beta j}(T-\tau)
$$

for all $j$ and rational $T>\tau$. It follows readily from (11.12) with $j=\beta$ that there is a subsequence $\left\{j_{\nu}\right\}=\left\{i_{a_{\nu}}\right\}$ of $\left\{i_{\nu}\right\}$ and a sequence of values $\left\{T_{\nu}\right\}$ of $T$, $T_{\nu} \downarrow \tau$, such that

$$
\lim _{\nu \rightarrow \infty} p_{j_{\nu} \beta}\left(T_{\nu}-r_{a_{\nu}}\right)=\lim _{\nu \rightarrow \infty} p_{\beta \beta}\left(T_{\nu}-\tau\right)=1 .
$$

This evidently contradicts condition $C(\beta)$. Thus if $C(\beta)$ is satisfied, $q_{\beta}<\infty$. The family of chance variables

$$
\left\{\frac{p_{j x(r)}(r-\tau)}{p_{\alpha x(r)}(r)}\right\}
$$

has, as we have seen, the property $\mathcal{E}^{*}$. Since these chance variables are nonnegative, there is convergence when $r \downarrow \tau$, with probability $1\left({ }^{26}\right)$. Since $x(r) \rightarrow x(\tau)$ in measure,

$$
\lim _{r \downarrow \tau} \frac{p_{\beta x(r)}(r-\tau)}{p_{\alpha x(r)}(r)}=\frac{1}{p_{\alpha \beta}(\tau)}
$$

almost everywhere where $x(\tau)=\beta$. We can suppose $\Lambda$ has been chosen so that (11.14) is true if $x(t) \in \Lambda$. Unless $x(r) \rightarrow \beta$ whenever $x(t) \in \Lambda, r \downarrow \tau$, and $x(\tau)=\beta$, there is an $x_{0}(t)$ in $\Lambda$ with $x_{0}(\tau)=\beta$, a sequence of integers $\left\{i_{\nu}\right\}$, and a sequence $\left\{r_{\nu}\right\}$ such that $x_{0}\left(r_{\nu}\right)=i_{\nu} \rightarrow \pm \infty, r_{\nu} \downarrow \tau$. Then, using (11.14),

$$
\frac{p_{\beta i_{\nu}}\left(r_{\nu}-\tau\right)}{p_{\alpha i_{\nu}}\left(r_{\nu}\right)} \rightarrow \frac{1}{p_{\alpha \beta}(\tau)}
$$

which contradicts $C^{*}(\beta, \tau)$. Thus if $C^{*}(\beta, \tau)$ is satisfied, $q_{\beta}<\infty$. (We are using here the fact which is implicit in the discussion of $\phi_{\beta}(h)$ above that if $x(\tau)=\beta$, $\lim _{r_{\downarrow} r} x(r)=\beta$ with probability 1 if and only if $q_{\beta}<\infty$.)

We shall need a somewhat stronger condition than $C^{*}(\beta, \tau)$ below. We shall say that condition $C^{* *}(\beta, \tau)$ is satisfied if $p_{\alpha \beta}(\tau)>0$ and if there are positive numbers $\eta, \delta$ such that if $0<s_{1} \leqq s_{2}<\delta, i \neq \beta, p_{\alpha i}\left(\tau+s_{1}\right)>0$, then

$$
p_{\beta i}\left(s_{2}\right)<\frac{p_{\alpha i}\left(\tau+s_{1}\right)}{p_{\alpha \beta}(\tau)}(1-\eta) .
$$

Condition $C^{* *}(\beta, \tau)$ is always satisfied in the finite-dimensional case, under hypothesis $H_{\alpha}$, if $\beta \in G_{\alpha}$ since (11.16) becomes (11.6) when $s_{1}$ and $s_{2}$ approach 0 .

(26) This is true if $r \downarrow \tau$ along any sequence of values (Doob, these Transactions, vol. 47 (1940), p. 460, Theorem 1.3) and this means the truth of the statement when $r \downarrow \tau, r \in R$ (Doob, these Transactions, vol. 42 (1937), p. 111, Theorem 1.3, or, in another formulation, Duke Mathematical Journal, vol. 4 (1938), pp. 758-759, Lemma 2). 
Theorem 12. Suppose that hypothesis $H_{\alpha}$ is true. Let $R$ be any denumerable everywhere dense $t$-set. Then there is a set $\Lambda$ of functions $x(t)$, of probability 1 , such that if $x(t) \in \Lambda$ the following statements are true.

(a) If the matrices are finite-dimensional, $x(r)(r \in R)$ has only isolated jumps as discontinuities.

(b) Either $\lim _{r \downarrow r}|x(r)|=\infty(r \in R)$, or there is an integer $\beta$, depending on the function $x(t)$ and on $\tau$, such that

$$
\lim _{r \downarrow \tau} \frac{x(r)-\beta}{1+|x(r)|^{2}}=0
$$

If condition $C(\beta)$ is satisfied, then either $\lim _{r \downarrow \tau}|x(r)|=\infty$ or there is an integer $\beta$ depending on the function $x(t)$ and on $\tau$, such that $\lim _{r \downarrow} x(r)=\beta(r \in R)$.

( $\left.\mathrm{b}^{*}\right)$ The statement of (b) remains true with $r \uparrow \tau$, instead of $r \downarrow \tau$, replacing condition $C(\beta)$ by $C^{* *}(\beta, \tau)$.

(c) For each $\tau$, there will be an integer $\beta$ (and $\beta=x(\tau)$ ), as described in (b), $\left(\mathrm{b}^{*}\right)$, with probability 1 .

Theorem 12 is closely related to work of Doeblin and Feller, with which it will be compared below.

The families $\left\{y_{t}\right\},\left\{y_{t}^{*}\right\}$ have the properties $\varepsilon, \varepsilon^{*}$, respectively. There is therefore an $\omega$-set $\Lambda$ of probability 1 , such that if $x(t) \in \Lambda$, the corresponding $y_{t},\left(y_{t}^{*}\right)$ coincide on $R$ with functions everywhere continuous on the right (left), for all $j$, rational $T, T^{*}$. (It has been proved above that there are no points of fixed discontinuity.) We can also suppose that $x(r) \neq j$ unless $p_{\alpha j}(r)>0$, if $x(t) \in \Lambda$. Then if $x_{0}(t) \in \Lambda$, and if $T>\tau>T^{*}>0, T, T^{*}$ rational, the following limits exist:

$$
\begin{aligned}
& \lim _{r \downarrow \tau} p_{x_{0}(r) j}(T-r), \\
& \lim _{r \uparrow \tau} \frac{p_{j x_{0}(r)}\left(r-T^{*}\right)}{p_{\alpha x_{0}(r)}(r)} .
\end{aligned}
$$

If $x_{0}(r)$ takes on a subscript $\beta$ for values of $r$ approaching $\tau$ from above, we can evaluate the limit in (12.2):

$$
\lim _{r \downarrow \tau} p_{x_{0}(r) j}(T-r)=\lim _{r \downarrow \tau} p_{\beta j}(T-r)=p_{\beta j}(T-\tau) .
$$

Then $\beta$ is uniquely determined, for if $\gamma$ had the same defining property, we should have $p_{\beta j}(T-\tau)=p_{\gamma j}(T-\tau)$ for all rational $T>\tau$, where $\beta, \gamma$ are both in $G_{\alpha}$. When $T \downarrow \tau$ this means (using hypothesis $H_{\alpha}$ ) that $\delta_{\beta j}=\delta_{\gamma j}$ for all $j$, impossible unless $\beta=\gamma$. Thus (12.1) is true. Then in the finite-dimensional case, $\lim _{r \downarrow \tau} x_{0}(r)=\beta$, that is, $x_{0}(r)=\beta$ for $r$ sufficiently near $\tau(r>\tau)$. In the infinite-dimensional case, unless $\lim _{r \downarrow \tau} x_{0}(r)=\beta$, we have proved that $\lim \sup _{r \downarrow r}\left|x_{0}(r)\right|=\infty$, and the method of proof of Theorem 11 can be car- 
ried through to find a contradiction to condition $C(\beta)$. We have now proved that Theorem 12(b) is true, supposing however that there is a subscript $\beta$ as described. (In the finite-dimensional case there is always such a $\beta$.) In the infinite-dimensional case, if there is no $\operatorname{such} \beta, \lim _{r \downarrow r}|x(r)|=\infty$. This finishes the proof of (b). The discussion when $r \uparrow \tau$ is carried on in the same way, using the existence of the limit in $\left(12.2^{*}\right)$. Theorem $12(\mathrm{a})$ is now obviously true. For a given $\tau, x(r) \rightarrow x(\tau)$ in measure, when $r \rightarrow \tau$, so there will be an integer $\beta$ as described above, and $\beta=x(\tau)$, with probability 1 .

As usual in this sort of discussion, instead of saying that $x(r)(r \in R)$ has the above described properties with probabiliy 1 , we could say that if a space $\Omega$ of a stochastic process is chosen properly, all the $x(t)$ in $\Omega$ will have the above properties, where $t$ ranges through all values.

Doeblin has considered a general Markoff process in which the transition probability of going from state $i$ at time $t$ to state $j$ at time $t^{\prime}$ is not supposed necessarily to be a function of $t^{\prime}-t$, and in which it is not supposed that the number of possible states is denumerably infinite. His hypotheses, when translated into our notation, and simplified because of the more special process being considered here, become

$$
\lim _{t \rightarrow 0} p_{i i}(t)=1
$$

uniformly in $i$. This hypothesis, combined with the hypothesis that the process is initially in state $\alpha$ is considerably stronger than hypothesis $H_{\alpha}$ (except in the finite-dimensional case, when, assuming hypothesis $H_{\alpha}$, Doeblin's condition is always applicable) and evidently also implies condition $C(\beta)$ for all $\beta \in G_{\alpha}$. Doeblin showed that under his hypotheses, and assuming some given initial state, neglecting an $\omega$-set of measure $0, x(r)(r \in R)$ has only isolated jumps as discontinuities $\left({ }^{27}\right)$.

Conversely, suppose that the process is initially in state $\alpha$, and that the $\omega$-measure has the property that $x(r)(r \in R$, a denumerable everywhere dense $t$-set) has only isolated jumps as discontinuities, with probability 1 . Theorem 11 shows that in this case there must be continuity at each fixed $\tau$, with probability 1 , that is, $q_{i}<\infty$ if $i \in G_{\alpha}$. Also, by Theorem $10, \sum_{j} q_{i j}=q_{i}$. Let $P_{i j}^{(n)}(t)$ be the probability $\left.{ }^{28}\right)$ that if $x(\tau)=i$ then $x(\tau+t)=j$ and $x(r)$ has $n$ jumps in going from $i$ to $j$, between $\tau$ and $\tau+t$. It is easily verified that if $i \in G_{\alpha}$

$$
\begin{aligned}
P_{i k}^{(0)}(t) & =\delta_{i k} e^{-q_{i} t} \\
P_{i k}^{(n+1)}(t) & =\sum_{j} \int_{0}^{t} P_{i j}^{(n)}(s) q_{j k} e^{-q_{k}(t-s)} d s \quad(n \geqq 0),
\end{aligned}
$$

(27) Skandinavisk Aktuarietidskrift, vol. 22 (1939), pp. 211-222.

(28) For this conditional probability to have a meaning we must suppose that $p_{\alpha i}(\tau)>0$; $\tau$ can always be so chosen, if $i \in G_{\alpha}$. 
and obviously

$$
p_{i j}(t)=\sum_{n \geqq 0} P_{i j}^{(n)}(t)
$$

Moreover if we suppose only hypothesis $H_{\alpha}$ to be true, and that $\sum_{j} q_{i j}=q_{i}<\infty$, $\left(i \in G_{\alpha}\right.$ ), then if $i \in G_{\alpha}$, considerations analogous to those used in the proof of Theorem 10 show that $P_{i j}^{(n)}(t)$ as defined in (12.5) will have the probability meaning described above. On the other hand, (12.6) is now true if and only if the only discontinuities of $x(r)$ are isolated jumps, with probability 1 . Feller has found necessary and sufficient conditions on the $q_{i}, q_{i j}$ that $(12.6)$ be true $\left({ }^{29}\right)$. The above remarks give a complete justification for Feller's probability interpretation (ibid., p. 498) of the $P_{i j}^{(n)}(t)$. (He did not need this interpretation in his proofs.) The details given in Theorem 12 on the character of $x(t)$ at a discontinuity which is not a jump round out Feller's description, arising from an entirely different background (ibid., pp. 512-513).

Suppose again that hypothesis $H_{\alpha}$ is satisfied and that $\sum_{j} q_{i j}=q_{i}<\infty$ for all $i \in G_{\alpha}$. The differential equations

$$
p_{i k}^{\prime}(t)=-q_{i} p_{i k}(t)+\sum_{j \neq i} q_{i j} p_{j k}(t) \quad\left(i \in G_{\alpha}\right)
$$

satisfied by the $p_{i j}(t)$, due to Kolmogoroff $\left({ }^{30}\right)$ are well known, but their intimate relation to the continuity properties of the $x(t)$ seems less well known. In fact, under the above hypotheses, we have shown (Theorem 10) that (with probability 1) if $x(\tau)=i \in G$, there is a first discontinuity of $x(r)(r \in R$, an everywhere dense denumerable $t$-set) after $\tau$, an isolated jump. Then the probability of going from $i$ to $k$ is the sum of the probabilities of going from $i$ to $j$ on the first jump, and then to $k$ (summed over $j$ ). Considerations analogous to those used in the proof of Theorem 10 now show that, evaluating the above probabilities,

$$
p_{i k}(t)=\sum_{j \neq i} \int_{0}^{t} e^{-q_{i}(t-s)} q_{i j} p_{j k}(s) d s+\delta_{i k} e^{-q_{i} t} .
$$

The equations of (13.1) are obtained by differentiating those of (13.2), in which the series can obviously be differentiated term by term. The second set of differential equations obtained by Kolmogoroff

$$
p_{i k}^{\prime}(t)=-q_{k} p_{i k}(t)+\sum_{j \neq k} p_{i j}(t) q_{j k}
$$

(29) These Transactions, vol. 48 (1940), pp. 506-507. Feller's results are applicable to considerably more general stochastic processes than those considered here.

(30) Mathematische Annalen, vol. 104 (1931), p. 429. Kolmogoroff imposed further restrictions on the $p_{i j}(t)$. Feller (op. cit., p. 495) obtained (13.1) with substantially our hypotheses given above. 
does not seem always to be true without further hypotheses. We shall show that the truth of (13.3) is equivalent to the imposition of certain regularity properties on the $x(t)$. The probability

$$
p_{i k}\left(t_{2}\right)-p_{i k}\left(t_{1}\right) e^{-q_{k}\left(t_{2}-t_{1}\right)} \quad\left(i \neq k, t_{2}>t_{1}\right)
$$

is at least equal to the probability that if $x\left(\tau-t_{2}\right)=i$, then $x(r)$ goes to $j$ at some point between $\tau-t_{1}$ and $\tau$, when $x(r)$ jumps to $k$, remaining at $k$ until $t=\tau$, summed over $j \neq k$. Thus

$$
p_{i k}\left(t_{2}\right)-p_{i k}\left(t_{1}\right) e^{-q_{k}\left(t_{2}-t_{1}\right)} \geqq \sum_{j \neq k} \int_{t_{1}}^{t_{2}} p_{i j}(s) q_{j k} e^{q_{k}(t-2-s)} d s .
$$

Moreover there is equality if and only if when $x(\tau)=i$, there is a last discontinuity of $x(r)$ before $\tau$, which is a jump, with probability 1. Dividing (13.4) by $t_{2}-t_{1}$ and letting $t_{2}-t_{1} \rightarrow 0$ we obtain

$$
p_{i k}^{\prime}(t) \geqq-q_{k} p_{i k}(t)+\sum_{i} p_{i j}(t) q_{j k}
$$

It is easily verified that (13.5) also follows directly from (0.1). Since there is equality in (13.5) if and only if there is equality in (13.4), we have obtained the following theorem.

THEOREM 13. Suppose that hypothesis $H_{\alpha}$ is satisfied and that $\sum_{j} q_{i j}=q_{i}<\infty$ for all $i \in G_{\alpha}$. Then (13.1) is always true; (13.3) is true (for all $t$ ) if and only if when $x(\tau)=i$, there is, with probability 1 , a last discontinuity of $x(r)$ before $\tau$, which is an isolated discontinuity (a jump).

It is interesting to note that if $p_{i j}(t)$ is the probability that if $x(\tau)=i$ then $x(\tau+t)=j$ and the transition from $i$ to $j\left({ }^{31}\right)$ is accomplished in a finite number of isolated jumps, then $p_{i j}(t)$ evidently satisfies $(0.1)$ except that $\sum_{i} p_{i j}(t)$ may be less than 1. Moreover (13.1) is also true for the $p_{i j}(t)$ since the derivation for the $p_{i j}(t)$ applies equally well to $p_{i j}(t)$. And the derivation we have given of (13.5), when applied to the $p_{i j}(t)$ actually gives equality: (13.3) is true of the $p_{i j}(t)$ in all cases. The latter fact was also proved by Feller.

(31) Strictly speaking, we should restrict $i, j$ to lie in $G_{\alpha}$.

UNIVERSITY OF ILLINOIS,

URBANA, ILL. 